\title{
Granular flow down a rough inclined plane: transition between thin and thick piles
}

\author{
Leonardo E. Silbert, James W. Landry, and Gary S. Grest \\ Sandia National Laboratories, Albuquerque, New Mexico 87185
}

\begin{abstract}
The rheology of granular particles in an inclined plane geometry is studied using molecular dynamics simulations. The flow-no-flow boundary is determined for piles of varying heights over a range of inclination angles $\theta$. Three angles determine the phase diagram: $\theta_{r}$, the angle of repose, is the angle at which a flowing system comes to rest; $\theta_{m}$, the maximum angle of stability, is the inclination required to induce flow in a static system; and $\theta_{\max }$ is the maximum angle for which stable, steady state flow is observed. In the stable flow region $\theta_{r}<\theta<\theta_{\max }$, three flow regimes can be distinguished that depend on how close $\theta$ is to $\theta_{r}$ : i) $\theta>>\theta_{r}$ : Bagnold rheology, characterized by a mean particle velocity $v_{x}$ in the direction of flow that scales as $v_{x} \propto h^{3 / 2}$, for a pile of height $h$, ii) $\theta \gtrsim \theta_{r}$ : the slow flow regime, characterized by a linear velocity profile with depth, and iii) $\theta \approx \theta_{r}$ : avalanche flow characterized by a slow underlying creep motion combined with occasional free surface events and large energy fluctuations. We also probe the physics of the initiation and cessation of flow. The results are compared to several recent experimental studies on chute flows and suggest that differences between measured velocity profiles in these experiments may simply be a consequence of how far the system is from jamming.

46.55.+d, 45.70.Cc, 46.25.-y
\end{abstract}

\section{INTRODUCTION}

The flow properties of granular materials puzzle engineers and physicists alike. Despite numerous experimental studies of heap flows, avalanches, and chute flows, there still does not exist a general consensus on the nature of typical granular flow. Early systematic experiments in inclined plane geometries were concerned with flow rates of granular material down chutest 3 , usually with smooth bases. Large regions of plug flow were observed, i.e. the whole bulk of the material slid down the plane at a velocity determined by the input mass rate and inclination angle of the chute. Kinetic theories for the inclined plane problem can explain some aspects of this flow behaviour for nearly elastic particles on smooth chutes胭. Other studies have determined structural features in the flow in two dimensional (2D) experiment 8 and simulations 8 . Still, there has not been the same success in predicting flows down rough inclines for inelastic, frictional particles.

Detailed measuring techniques have been developed recently to capture various flow properties of granular material down rough inclined planes 9 and in heap flow geometries 12 t 4 . Accompanying these more thorough experiments, further theoretical treatments have also emerged 100.56 , together with full three dimensional (3D) computer simulations 192 . Despite this growth in literature, there appears to be a disturbing feature of these studies: reports on quantities such as velocity profiles differ quite dramatically. While some reports are agreement with the pioneering results of Bagnold 23:24, others are not.

In 1954, Bagnold23 proposed a momentum transfer picture to describe his experimental results on inertial granular flow. This simple 2D model describes the collisional process between particle layers during flow. The resulting relations 
predict a shear stress $\sigma$ that is proportional to the square of the strain-rate,

$$
\sigma \propto \dot{\gamma}^{2}
$$

where the strain-rate is defined as the derivative of the velocity in the direction of flow $(x)$ with respect to depth $(z)$, $\dot{\gamma} \equiv \partial v_{x}(z) / \partial z$. Typically, in local, Newtonian, hydrodynamic treatments of granular dynamics 17 , the relationship $\sigma=\eta \dot{\gamma}$ is used, combined with the kinetic term $\eta \propto T^{1 / 2}$, where $\eta$ is a viscosity and $T$ is the granular temperature (to be discussed in section III B). Since the strain-rate is also proportional to the square root of the granular temperature $\dot{\gamma} \propto T^{1 / 2}$, Eq. 目is then recovered. Bagnold's argument, applied to the case of bulk granular flow, is predicated on a constant density depth-profile with no-slip condition at the base. This leads to a velocity depth-profile of the form,

$$
v_{x}(z) \propto\left[h^{3 / 2}-(h-z)^{3 / 2}\right],
$$

for a pile of total height $h$, where $z$ is measured from the bottom of the pile. Because Bagnold scaling comes naturally from dimensional analysis in the absence of intrinsic time scales, one might expect that most studies would observe this kind of rheology. However, results depend strongly on the experimental procedure; profiles of this form have only been reported in a few instances $\frac{6}{\text {. }}$.

One method to study gravity-driven flows is to construct a wedge-shaped static pile that evolves to produce an inclined surface close to the angle of repose of the material12 14, Fig. 11(a). We term these heap flows. Here, the inclination of the surface is determined by material properties. To study flow, material is supplied via a hopper-feeder mechanism at the high end of the pile and flow is induced at the free surface. In this case, although the flow rate may be varied by changing the discharge rate of the hopper, the system typically flows only in a thin surface layer, indicated by the shaded region in Fig. 1 1 (a), which is of order 10 particles diameters in depth. In one such experiment, Lemieux and Durian 12 observed avalanche behavior for low particle flux, characterized by sporadic downstream movement at the free surface. On increasing the particle flux, a velocity profile with depth was observed to be approximately exponential in the thin surface layer. More recently, utilizing a similar experimental geometry, Komatsu et al.13 found that the 'stationary' bulk material beneath the surface flow actually undergoes very slow, 'creeping' motion with a well-defined exponential velocity profile at longer times.
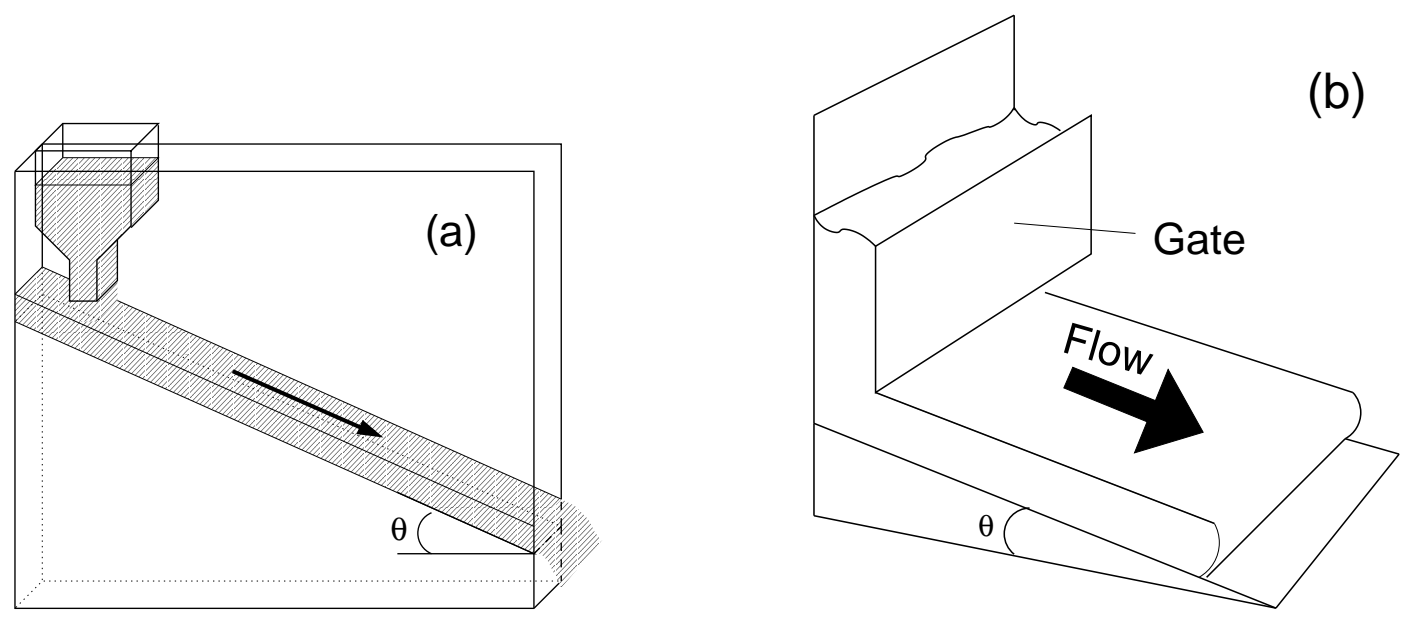

FIG. 1. Schematics of experiments used to study gravity-driven, granular flows. The (a) heap flow geometry used by Lemieux and Durian 12 and Komatsu et al.13, and (b) the inclined plane geometry employed by Pouliquen for chute flow studies 9 . Our simulations correspond closely to the Pouliquen experiments. 
Another method uses inclined planes to study flows and avalanches, typically with rough beds designed to reduce slip at the base, thereby avoiding plug or unstable flow 2525 . We term these chute flows. Roughness is introduced onto the chute by gluing particles to the chute base 1.2 .27 , or by attaching a frictional material to the base, such as the velvet cloth used by Daerr and Douady28. Chute flow studies of two and three dimensional systems with rough bases report depth-averaged velocities with both linear 10.11 and non-linear dependence 911 on height. The geometry for these experiments is shown in Fig. 国(b). The material is contained in a reservoir at one end of a plane inclined at a variable angle $\theta$ with respect to the horizontal. After the gate to the reservoir is opened, material flows down the incline. Steady flow occurs only at high enough angle; while flows come to rest quickly on the rough bed for small inclination angles 29. Increasing the gate opening leads to thicker flows down the incline: there are no dead zones along the incline and all the material flows down the chute. This allows one to independently control the inclination angle and material flux of the pile, in contrast to the heap experiments.

Using a range of inclination angles and flow depths, Pouliquen 9 measured the depth-averaged flow velocity of material for piles of different thickness. The velocity in the direction of flow scaled with height of the pile as $v_{x} \propto h^{3 / 2}$, which has a different form from the heap flow results reported by Lemieux and Durian日2 and Azanza et al.10. However, Ancey's comprehensive experimental investigation 11 demonstrated that during the course of one experiment, a range of flow behaviors is obtained that depends, among other parameters, on the flow rate of the material. Ancey's results suggest that the different flow profiles observed are not necessarily in contradiction, but may be a consequence of the conditions under which the measurements are made.

In our earlier studies21.22, we simulated the rheology of dense, cohesionless, frictional spheres in inclined plane geometries. Employing periodic boundary conditions in the flow and vorticity directions, our simulations can be considered equivalent to the experimental set up in Fig. 1 (b), for an infinitely long chute far away from the material reservoir and side walls, with a continuous supply of material. Our previous study was concerned with the flow of thick piles on rough bases over a range of tilt angles where we found Bagnold scaling in stable steady flow, for a range of parameters (e.g. particle friction and inelasticity, and inclination angles).

To investigate the discrepancies between the various experimental results previously mentioned, we have extended our previous computational studies of granular chute flows to cover a wide range of pile thickness, from very thin to very thick piles, both near and far from the angle of repose $\theta_{r}$. We show here that even thick piles experience a crossover in behavior from Bagnold rheology to linear velocity profiles, and also avalanche-like flow near the angle of repose. We therefore refine our definition of stable, steady state flow: we report on three distinct steady state flows observed in our simulations which match the experimentally observed flows reported by Lemieux and Durian 12 (avalanches), Ancey11 (linear velocity profiles), and Pouliquen' (Bagnold scaling). Our main conclusion is that the observed behavior is strongly dependent on how close the system is to jamming or how close $\theta$ is to $\theta_{r}$.

In the next section we briefly describe the simulation procedure. In section III] we present the results of our simulations, in particular defining the phase space of our study and describing transitions between the flow properties of thick and thin piles both near and far from the angle of repose. We also present results for our analyses on the initiation and cessation of flow in section IV. In section $\mathrm{V}$ we summarize the main conclusions of this work. 


\section{SIMULATION PROCEDURE}

Here we briefly introduce the simulation model; a thorough description of the technique is available elsewhere 2122. We model $N$ cohesionless, frictional, and inelastic spheres of diameter $d$ and mass $m$ flowing on a fixed, rough bed of area $A$ with a free top surface. Although most of our results are for monodisperse systems, we have verified that our results hold for systems with small polydispersity 30 .

We employed a 3D contact force model that captures the major features of granular particles: interactions between spheres are modeled as Hertzian contacts with static friction. The implementation of the contact forces, both the normal forces and the shear (friction) tangential forces, is essentially a reduced version of that employed by Walton and Braun31, developed earlier by Cundall and Strack 32 . Normal and tangential forces acting on particle $i$ due to contact with particle $j$ are given by

$$
\begin{aligned}
& \mathbf{F}_{n_{i j}}=\sqrt{\delta_{i j} / d}\left(k_{n} \delta_{i j} \mathbf{n}_{i j}-m_{\mathrm{eff}} \gamma_{n} \mathbf{v}_{n_{i j}}\right), \\
& \mathbf{F}_{t_{i j}}=\sqrt{\delta_{i j} / d}\left(-k_{t} \mathbf{u}_{t_{i j}}-m_{\mathrm{eff}} \gamma_{t} \mathbf{v}_{t_{i j}}\right),
\end{aligned}
$$

where $\delta_{i j}$ is the particle-particle overlap, $\mathbf{n}_{i j} \equiv \mathbf{r}_{i j} / r_{i j}=\left(\mathbf{r}_{i}-\mathbf{r}_{j}\right) /\left|\mathbf{r}_{i}-\mathbf{r}_{j}\right|$, is the unit normal along the line of centers, for particle $i$ situated at $\mathbf{r}_{i}$ and $j$ at $\mathbf{r}_{j} . \mathbf{v}_{n, t}$ are relative velocities in the normal and tangential directions respectively, and $\mathbf{u}_{t_{i j}}$ is the elastic tangential displacement that is set to zero at the initiation of a contact. $m_{\text {eff }}$ is the effective mass and $k_{n, t}$ and $\gamma_{n, t}$ are elastic and viscoelastic constants respectively. Thus in a gravitational field $\mathbf{g}$, the force on particle $i$ arises from Newton's second law for the translational and rotational degrees of freedom,

$$
\begin{aligned}
\mathbf{F}_{i}^{\mathrm{tot}} & =m \mathbf{g}+\sum_{j} \mathbf{F}_{n_{i j}}+\mathbf{F}_{t_{i j}} \\
\boldsymbol{\tau}_{i}^{\mathrm{tot}} & =-\frac{1}{2} \sum_{j} \mathbf{r}_{i j} \times \mathbf{F}_{t_{i j}},
\end{aligned}
$$

and the force on particle $j$ is determined from Newton's third law.

As shown in Fig. 2, we define flow to be parallel to the $x$-direction, $z$ the height normal to the base, and $y$, the vorticity axis, is normal to the $x z$-plane. Values for the friction coefficient $\mu$, and the elastic and viscoelastic coefficients (which are related to the moduli of the material) are chosen to be characteristic of real materials. For all simulations in this study we set $\mu=0.50, k_{n}=2 \cdot 10^{5} \mathrm{mg} / d, k_{t}=\frac{2}{7} k_{n}, \gamma_{n}=50 \sqrt{\mathrm{g} / \mathrm{d}}$, and $\gamma_{t}=\gamma_{n} / 233$. The time-step $\delta t=10^{-4} \tau$, where $\tau=\sqrt{d / g}$. For a millimeter sized particle $\tau \approx 0.01 \mathrm{~s}$. All simulations were run for at least $1 \cdot 10^{7} \delta t$ before any quantities were measured.

Figure 2 shows snapshots of flowing systems where the area of the chute base $A \equiv L_{X} \times L_{Y}=20 d \times 10 d=200 d^{2}$, and we vary the total number of particles $N$. The fixed chute bed is made from a random close packing of particles with the same diameter as those in the bulk, mimicking gluing particles onto a chute base as in Ref. 0 , for example. In section IIIB we kept $A=200 d^{2}$ fixed. In section [V], we used a base with dimensions $A=200 d \times 10 d=2000 d^{2}$. We have shown 22 that results for thick piles are robust with respect to the dimensions of the chute base. The height of a static pile $h_{\circ}=N / A \rho_{\circ}$ depends on the density of the static packing $\rho_{\circ}$, or equivalently the volume fraction $\phi_{\circ} \equiv \rho_{\circ} \pi / 6(\approx 0.6022)$ which is defined in the hard sphere limit (where the subscripts denote the static values). In the flowing state, the actual height of the pile increases with inclination angle due to dilation effects of the flow. However, 
the dependence of $h$, and therefore $\rho$, on $\theta$ is weak. We find that the computed bulk volume fractions vary over a small range, $0.55 \lesssim \phi \lesssim 0.60$, for steady state flows for the particular parameters chosen in this paper22. Thus for the simulations $h_{\circ}=\pi N / 6 A \phi_{\circ}$ : for $N=5000, h_{\circ} \approx 22 d$.

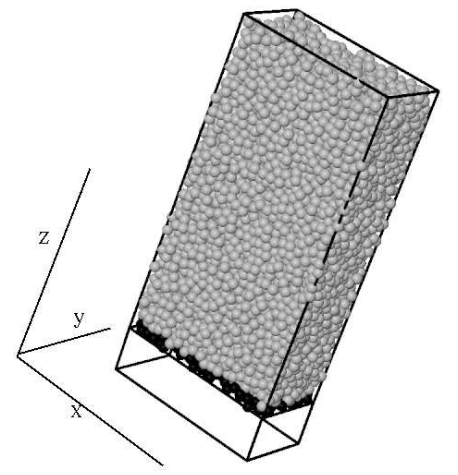

(a)

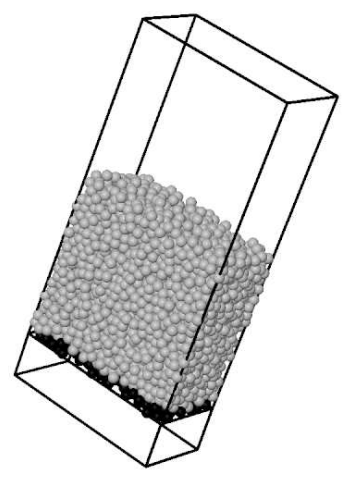

(c)

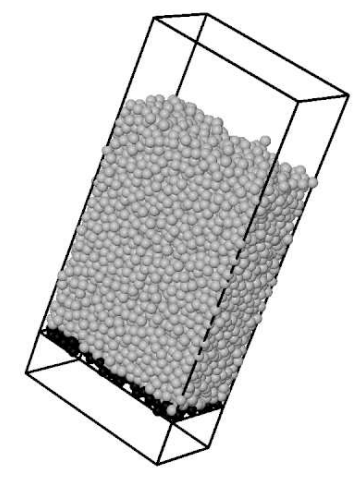

(b)

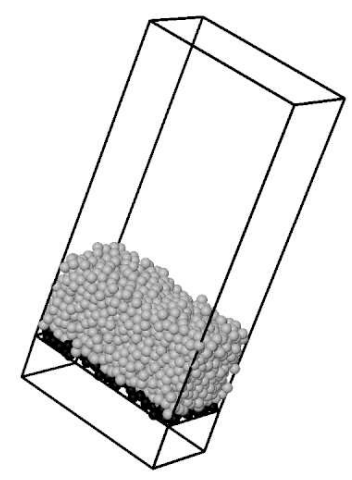

(d)

FIG. 2. Configuration snapshots for systems of various sizes with a chute base of fixed area $A=200 d^{2}$, that is inclined at an angle $\theta=24^{\circ}$ to the horizontal, where (a) $N=8000$, (b) $N=6000$, (c) $N=4000$, and (d) $N=2000$. The fixed rough base is denoted by the black particles, the flowing particles are grey, and the frame is a guide to the eye fixed at the height $h \approx 39.6 d$ of the $N=8000$ system to provide a comparison between the different flow heights.

\section{RESULTS}

In our previous studies2122, we roughly determined the range of parameters where steady state flow existed for chute flows, focusing mainly on steady state flow for thick piles away from $\theta_{r}$. We found that $\theta_{r}$ is approximately independent of height for piles of sufficient depth. In this work we perform a more thorough analysis of the entire region of the $h-\theta$ phase space. We determine several different flow regimes within the stable, steady state region which are qualitatively identical to several experimental observations in inclined plane geometries, on avalanche dynamic, 28,34 
and rheology 1 12. Although the exact location of the boundaries of the phase picture shown in Fig. 3 are dependent on specific particle parameters, such as the friction and damping coefficients, the features we report here are general to systems that exhibit steady state flow.

\section{A. Phase Behavior}

The overall phase behaviour is determined by the height of the pile $h$ and the angle of inclination $\theta$, as characterised in $2 \mathrm{D}$ by Pouliquen and Renault 29. A prominent feature of that study is the existence of a flow line, later observed in 3D experiments by Pouliquen $\mathrm{O}$ and also in studies of avalanches 28 . The flow line defines the angle of repose as a function of height of material flowing $\theta_{r}(h)$. One can equivalently refer to this in terms of the conjugate variable $h_{\text {stop }}(\theta)$ : $\theta_{r}(h)$ is the angle at which a pile of thickness $h$ will cease to flow, and conversely, $h_{\text {stop }}(\theta)$ gives the maximum thickness of material that will cease to flow at an inclination angle $\theta$. Various theoretical treatments have also successfully predicted the existence of the flow line 18,8 . Experimenta 27,28 and numerical 36 studies have also identified distinct sub-regions within the stable flow regime of which more will be discussed below.

In order to quantify this boundary with precision for our system, we performed a number of simulations to generate the $h-\theta$ phase diagram shown in Fig. 局. Initially, a static packing with a volume fraction $\phi \approx 0.60$, at $\theta=0^{\circ} 87$, is tilted until flow is observed. The maximum angle of stability $\theta_{m}$ is defined as the angle at which flow is initiated and largely depends on construction history of the initial static packing, such as the roughness of the supporting bed and the packing fraction of the initial configuration 38 . The angle of repose $\theta_{r}$ is defined as the inclination angle at which the system subsequently jams and flow ceases upon reducing $\theta$ back down.

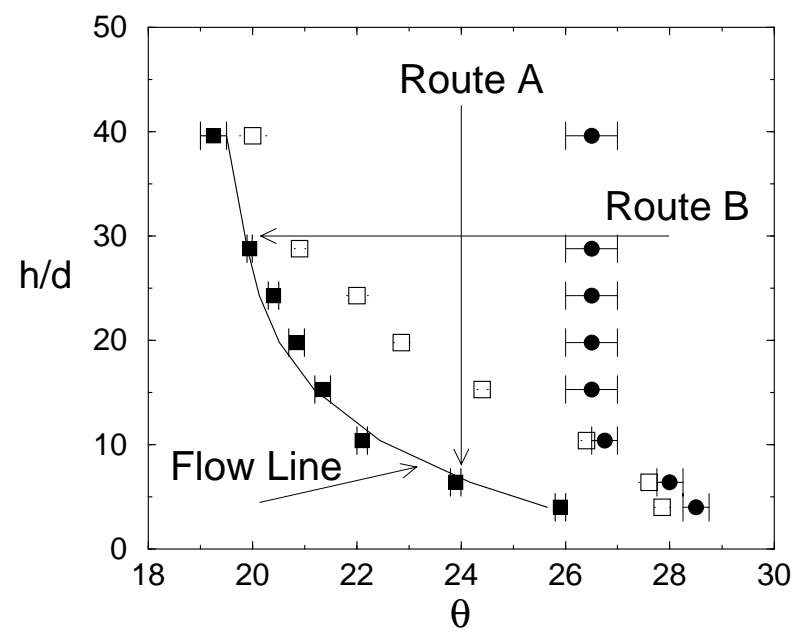

FIG. 3. The $h-\theta$ phase diagram for granular chute flow, where $h$ is the height of the pile. The filled squares are the simulation data for $\theta_{r}(h)$ and we indicate the flow line as a fit to Eq. 6 . The open squares are $\theta_{m}(h)$. Solid circles are $\theta_{m a x}(h)$, the angle at which we observe the onset of unstable flow. Route A corresponds to a path taken by keeping the angle fixed and reducing the system size $N$, whereas Route B has $N$ fixed and decreasing inclination. These routes are discussed in section IIIB.

Our initial packing was generated by taking a flowing system and gradually reducing the inclination down to $0^{\circ}$ and then allowing the system to relax until it became completely static. Experimental studies on avalanches have shown 
that $\theta_{m}$ can fluctuate over a range of $3^{\circ}-4^{\circ}$ for different packings with the same height because of construction history effects27.

We have also observed avalanche-like dynamics in our simulations. We characterise avalanches as distinct from regular or continuous flow by observing the time evolution of bulk quantities of the system such as bulk kinetic energy or stress. For continuous flow, the stress or bulk energy will fluctuate about a well-defined mean value, with fluctuations typically less than $10 \%$ of the mean. In the case of avalanches, the stress and energy fluctuate about a mean value with $\mathrm{O}(1)$ fluctuations, indicative of sporadic flow that mainly occurs at the free surface. We also checked any possible system size dependence. To compare with our smaller systems, we studied a system with $N=32000$ and $A=80 d \times 40 d$ (i.e. approximately $10 d$ high on a base with an area four times our standard system size). We again observed avalanche-like behaviour for the big system, although we cannot rule out that in the limit of infinite system size, avalanches might not be observed.

From our extensive study, we were able to determine the following general features of flow down inclined planes: there are three principal regions, corresponding to no flow, stable flow, and unstable flow. For a pile of given thickness $h$, and fixed microscopic interaction parameters, these three regions are separated by two angles: $\theta_{r}$, the angle of repose, and $\theta_{\max }$, the maximum angle for which we observe stable flow.

No flow $\theta<\theta_{r}$ : the system does not flow. Starting from $\theta=0^{\circ}$, an inclination of $\theta=\theta_{m}>\theta_{r}$ is required to initiate flow. As $\theta_{m}$ is approached, a few particles at the free surface rearrange and tumble short distances before coming to rest, but this does not lead to bulk motion or flow 39 . $\theta_{r}$ is the angle at which flow ceases on reducing the inclination from a flowing state - it is the lowest angle at which flow is observed and depends on material properties (friction and inelasticity) 40 , as well as the roughness of the supporting bed.

Stable flow $\theta_{r}<\theta<\theta_{\max }$ : bulk flow is observed (after flow is initiated). The bulk averaged kinetic energy of the system is constant with time and the entire pile is seen to be in motion while no slip is observed at the bottom with these rough boundary conditions. The stable flow region can be divided into the following three regimes:

Continuous flow I $\theta>>\theta_{r}$ : away from the flow line, the rheology is well approximated by Bagnold scaling, Eqs. (11) and (2).

Continuous flow II $\theta_{r}<\theta \lesssim \theta_{m}$ : close to the flow line, the rheology is not Bagnold-like, rather the velocity profile is better approximated by a linear relationship with depth $v(z) \propto z$. The upper boundary $\theta_{m}$ is also seen in experimental studies of avalanches and differentiates between different flow mechanisms, depending on whether one is above or below $\theta_{m} 28$.

Avalanching flow $\theta \approx \theta_{r}^{+}$: Very close to the angle of repose, but just above it, regular continuous flow is not observed, rather in this regime, steady state involves slow, underlying creep motion of the entire pile combined with sporadic free surface motion down the incline. In the vicinity of $\theta_{r}$, extremely large energy fluctuations are observed 11 .

Unstable flow $\theta>\theta_{\max }$ : the development of a shear thinning layer at the bottom of the pile results in lift-off and unstable acceleration of the entire pile. $\theta_{\max }$ becomes approximately constant for $h \gtrsim 10 d$.

The flow descriptions above capture the principal observations of several different experiments of gravity-driven flow 91 12 2. In particular, the transition from no flow to avalanche flow at $\theta \approx \theta_{r}$ is similar to the observations 
reported by Lemieux and Durian 12 for low material flux. The transition from avalanches to our continuous flow II regime appears to resemble the observations reported by Daerr and Douady 28 of avalanche studies for $\theta_{r}<\theta<\theta_{m}$. Similar observations have been reported for rotating cylinder experiments 42 .

Pouliquen, later verified by Daerr and Douady 28 , characterized the flow line - the dividing line between no flow and flow. Using an empirical argument, Pouliquen suggested the following relation:

$$
\tan \theta_{r}=\tan \theta_{1}+\left[\tan \theta_{2}-\tan \theta_{1}\right] \exp \left(-\frac{h}{l}\right),
$$

where $\theta_{1}$ is the angle at which $\theta_{r}$ becomes independent of depth, $\theta_{2}$ is the largest value of $\theta_{r}$, and $l$ is a characteristic length scale. In Fig. 3 we fit Eq. 目 to our simulation points and obtained the following fitting parameters; $\theta_{1}=$ $19.60^{\circ}\left(19.55^{\circ}\right), \theta_{2}=28.50^{\circ}\left(27.75^{\circ}\right)$, and $l \approx 8.5 d$, where the values in parentheses are the data points from the simulations. Our simulations not only predict the phase behavior of chute flows, but also capture the subtle features emerging in recent experiments. In the following sections we present various depth profiles of density, velocity, and stress to better quantify the transitions between thin and thick piles.

\section{B. Depth Profiles}

We computed depth profiles as a function of $z$ for the packing fraction $\phi$, velocity in the direction of flow $v_{x}$, strain-rate $\dot{\gamma}$, shear stress $\sigma_{x z}$, and granular temperature $T$ (see definition below), for a range of system sizes and inclination angles. Since our simulations employ periodic boundary conditions in the plane perpendicular to height, our simulations are not influenced by the effects of side walls and are therefore equivalent to measurements made along the centre line of a real chute flow, away from the edges. It has been recognised that profiles taken at the side walls are significantly different from those made along the centre line112.20.

Because of the dependence of $\theta_{r}$ on $h$, we approached $\theta_{r}$ in two ways: fixing the inclination angle of the chute and observing flow in systems of different heights until flow ceased for a pile of height $h_{\text {stop }}(\theta)$ (route A). Alternatively, we held $N$ fixed and reduced the inclination angle of a flowing state down to $\theta_{r}(h)$ (route B).

Route A: Figures 4 团 show various bulk profiles for route A, that is, systems at a fixed inclination angle of $\theta=24^{\circ}$ but of varying sizes, $N=8000,5000,3000,2000,1000$. The density profiles in Fig. 团 exhibit a constant density region throughout the bulk of the material which is a familiar observation for thicker systems2.

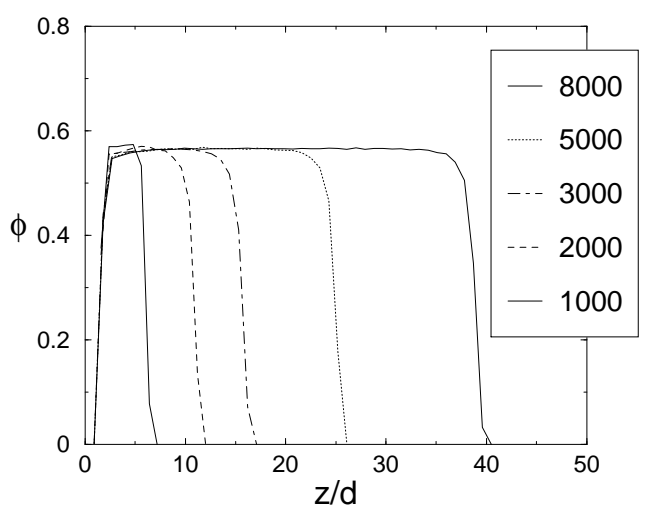

FIG. 4. Depth profiles of the packing fraction $\phi$, for systems of various size $N$, varying in heights from $h \approx 40 d$ to $5 d$ for $\theta=24^{\circ}$ : route A in Fig. $\mathrm{B}$. 
The flow velocity $v_{x}$ and strain-rate $\dot{\gamma}$ profiles shown in Fig. 5 are for the same systems reported in Fig. 目. For moderate to thick piles, $h \gtrsim 15 d$, the velocities and corresponding strain-rates scale with height. However, for $h \lesssim 15 d$, there are significant differences. The velocity profiles, top panel in Fig. 同, for the thinner piles at first become more linear with depth, then ultimately have a curvature opposite to that of the thicker piles. This gradient transformation is more apparent from the strain-rate curves (bottom panel Fig. [): for thicker piles $\dot{\gamma}$ decreases from the chute base toward the free surface of the flowing pile, whereas in thinner piles there is a range of system sizes where $\dot{\gamma} \approx$ constant throughout the pile, leading to a linear velocity profile. For thinner systems still, $\dot{\gamma}$ increases near the free surface.

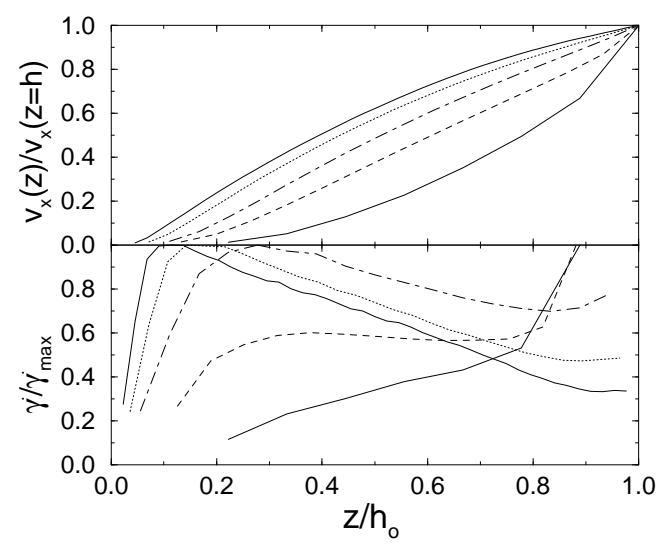

FIG. 5. Depth profiles of the velocity in the direction of flow (top panel) and the corresponding strain-rate (bottom panel), each normalized by their maximum value, for various system sizes for $\theta=24^{\circ}$. Note the depth is normalized by the respective height for clarity. Legend as in Fig. 6

In this work, we define the "granular temperature" $T$ to be the averaged velocity fluctuations about the mean bulk flow velocity at that height, $T \equiv\left\langle v^{2}\right\rangle-\langle v\rangle^{2}$. The temperature in the flow direction is defined as $T_{x}=\left\langle v_{x}^{2}\right\rangle-\left\langle v_{x}\right\rangle^{2}$. Figure 6 shows the change in the profiles of $T_{x}$ from thick to thin piles, which is similar to the behavior of $\dot{\gamma}$. From the simulations we find that for all sizes,

$$
T_{x} \propto \dot{\gamma}^{2}
$$

although the data for thinner piles is considerably noisier.

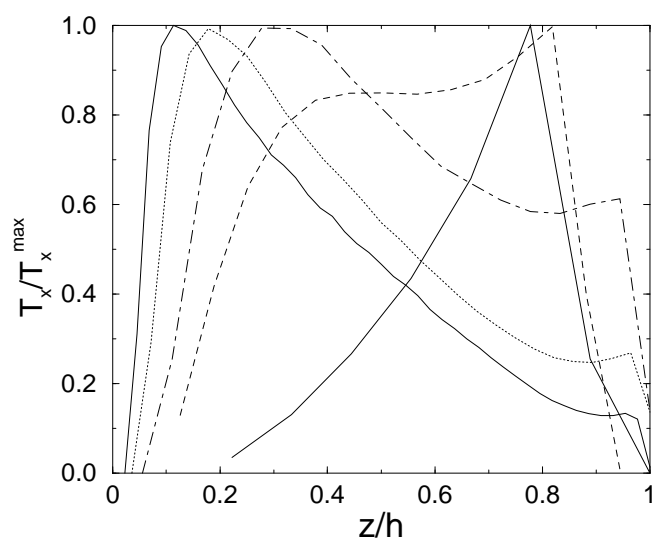

FIG. 6. Profile of the granular temperature $T_{x} / T_{x}^{\max }$, in the direction of flow as a function of $z$ for various system sizes for $\theta=24^{\circ}$. Legend as in Fig. 6 . 
Similarly, the rheology plot in Fig. 0 shows that thicker piles display Bagnold rheology: $\sigma_{x z} \propto \dot{\gamma}^{2}$, but there is a transition away from this as the piles become thinner. For thin piles, $5 \lesssim \frac{h}{d} \lesssim 15$, the strain rate is approximately constant away from the free surface and the chute base. For the thinnest piles $h \approx 5 d$, the strain rate decreases with shear stress. This may come about from competing length scales in the system, i.e. the thickness of the flow determines the separation between the top free surface and the chute base. Some theoretical treatments based on these considerations have been used to explain the differences between observed velocity profiles in flowing piles 16 .

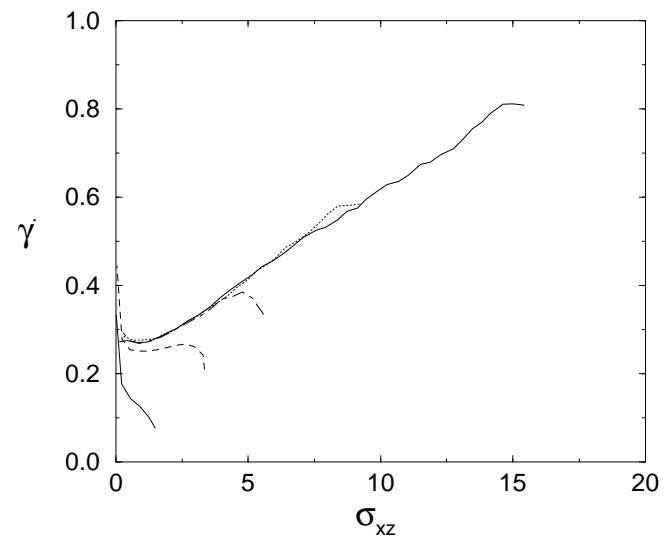

FIG. 7. Rheology data for various system sizes for $\theta=24^{\circ}: \dot{\gamma}$ vs. $\sigma_{x z}$. Legend as in Fig. 4 .

Route B: Figures 811 show depth profiles for a system taken along route B in Fig. 3. These are for a system of fixed size $N=6000$, where we vary the inclination angle down toward $\theta_{r}$. In Fig. 8 the density profiles for the system $N=6000$ are shown for a range of inclination angles $\theta=25^{\circ}, 23^{\circ}, 22^{\circ}, 21^{\circ}, 20^{\circ}$. We see the constant density region, where the bulk density decreases with increasing angle due to dilatancy effects. However, there are no qualitative differences between the behaviour at different angles.

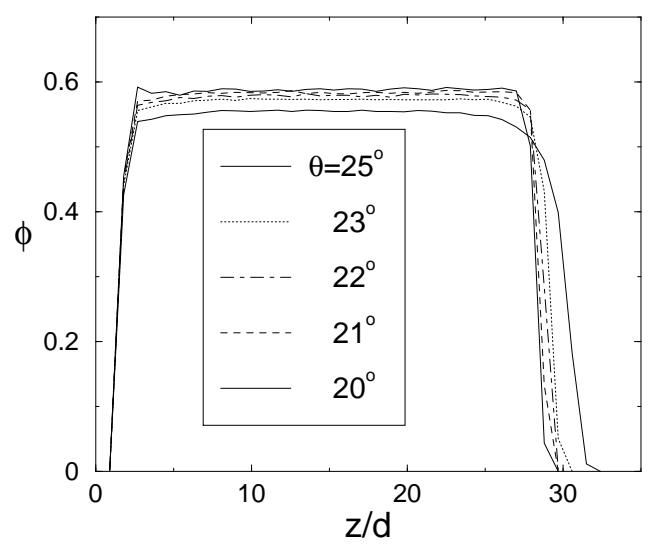

FIG. 8. Depth profiles of the packing fraction $\phi$, for a system with a fixed number of particles, $N=6000$, over a range of inclination angles, route B in Fig. 3 .

For the velocity and strain-rate profiles shown in Fig. 9, differences do emerge, and these differences mimic the behavior observed along route A. Away from the flow line, the velocity profiles scale as Eq. 2, whereas for systems at lower angles the velocity profiles change to linear. $\dot{\gamma}$ changes likewise as does the granular temperature (shown in 
Fig. 10).

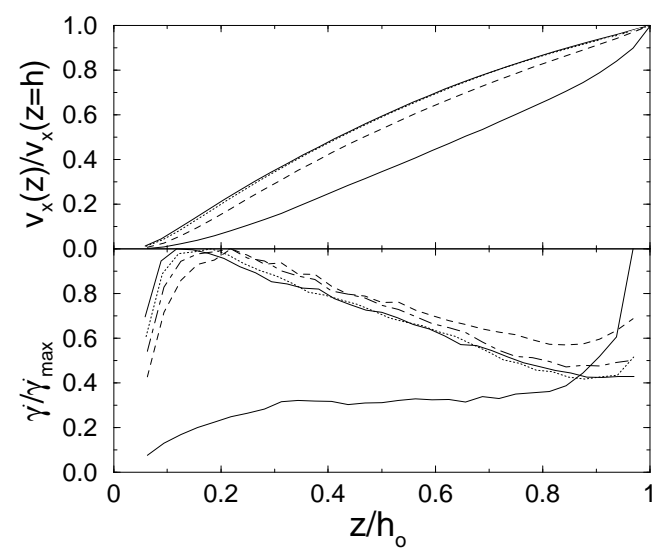

FIG. 9. Profiles for the velocity in the direction of flow $v_{x}$ (top panel) and the corresponding strain-rate $\dot{\gamma}$ (bottom panel), each normalized by their respective maximum values, for a system with a fixed number of particles, $N=6000$, over a range of inclination angles inclination angle. The height is normalized by the system size. Legend as in Fig. 目.

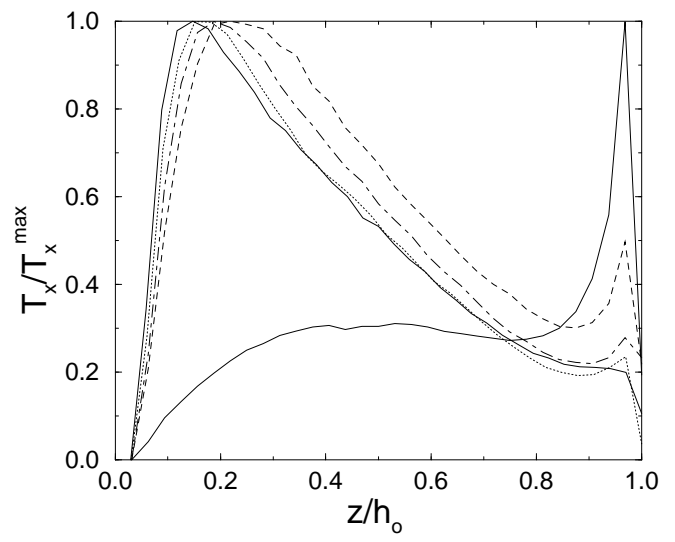

FIG. 10. Profile of the x-component of the granular temperature $T_{x} / T_{x}^{\max }$ for a system with a fixed number of particles, $N=6000$, over a range of inclination angles. Legend as in Fig. 8 .

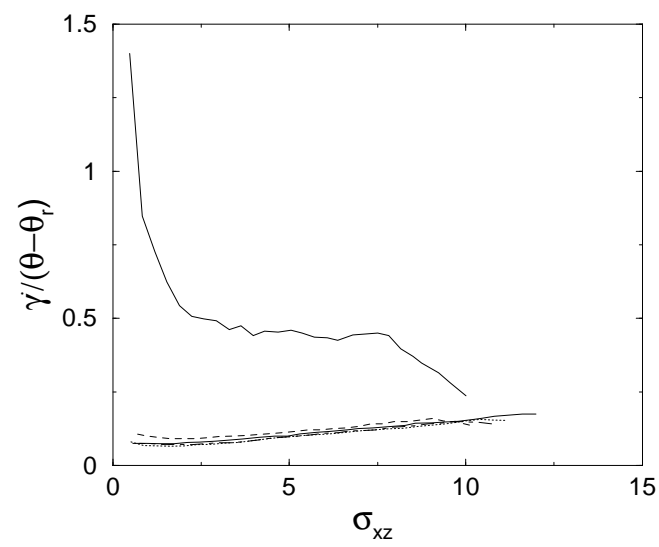

FIG. 11. Rheology data for a system with a fixed number of particles $N=6000$, over a range of inclination angles: $\dot{\gamma}$ normalized by $\left(\theta-\theta_{r}\right)$ vs. $\sigma_{x z}$. Legend as in Fig. 目. 
In Fig. 11 we plot the rheology curves for the different angles. We have rescaled $\dot{\gamma}$ by $\left(\theta-\theta_{r}\right)$, for convenience. We find that for systems with $\left(\theta-\theta_{r}\right) \gtrsim 1.0^{\circ}$, the rheology plots scale well. However, for the system that is closest to $\theta_{r}$, the flow behavior is very different, though similar to the differences observed along Route A.

Therefore the two routes A and B indicated in Fig. 3 offer complementary paths to similar states. Because of the very nature of the dependence of $\theta_{r}$ on $h$, the flow properties of systems at different angles and different heights show similarities provided they are taken equivalently close to $\theta_{r}$.

\section{Velocity Scaling}

Pouliquen 1 showed that flows of thick and thin piles approximately obey a simple scaling relation. Nondimensionalizing $v$, the velocity measured along the centre line at the top of the flowing pile by $\sqrt{g h}$, where $\mathrm{g}$ is the acceleration due to gravity and $h$ is the measured height of the pile, one obtains the Froude number $F r \equiv v / \sqrt{g h}$. By plotting the Froude number against $h / h_{\text {stop }}$, where $h_{\text {stop }}(\theta)$ is the height of a pile at which a system flowing at an angle $\theta$ would stop, Pouliquen was able to collapse his full data set of systems with different particle size and properties. Pouliquen proposed the simple scaling relation,

$$
\frac{v}{\sqrt{g h}}=\beta \frac{h}{h_{\text {stop }}} .
$$

where the coefficient $\beta$ was fit to the experimental data, resulting in an apparently universal value, $\beta_{\exp }=0.136$.

The quantity $h_{\text {stop }}$ encodes details of the particle roughness, the base roughness and all other intrinsic properties of a particular system. Accordingly, one need only measure $h_{\text {stop }}$ of the system in question to determine the flow properties. In a similar vein, in Fig. 12 we plot the flow velocity, taken from the top of the flowing pile, non-dimensionalised by $\sqrt{g h}$ for each height $h$ against $h / h_{\text {stop. }}$. The data agrees well with a linear relation, giving a coefficient $\beta_{\text {sim }}=0.147$, which is in good agreement with the experimental result determined by Pouliquen, $\beta_{\exp }=0.136$.

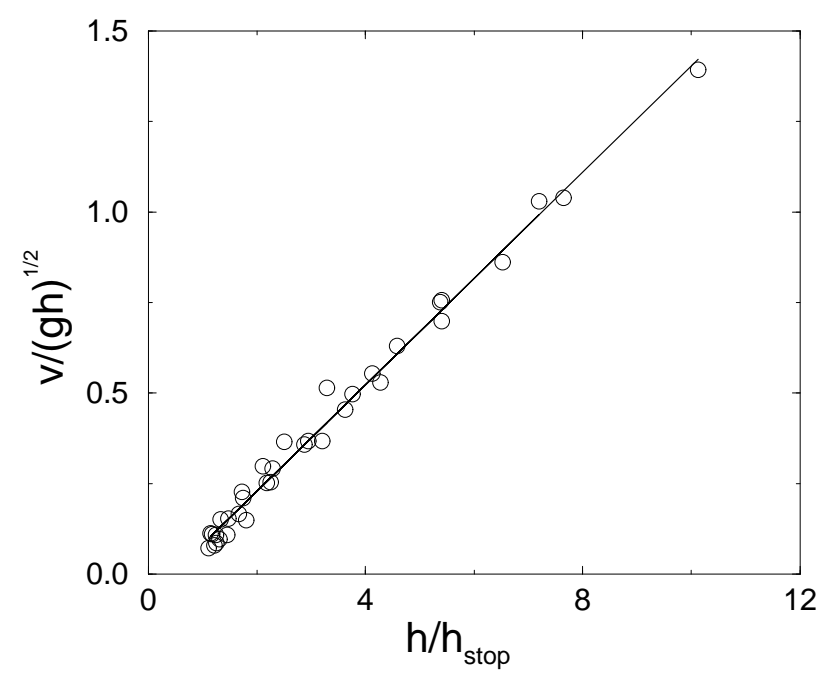

FIG. 12. Froude number $v / \sqrt{g h}$ as a function of $h / h_{\text {stop }}$ for different inclinations. A measure of the slope gives $\beta_{\text {sim }}=0.147$, compared with Pouliquen's experimental determination of $\beta_{\text {exp }}=0.136$. 
The scaling relation Eq. 9 although simple, appears to be quite robust. Pouliquen 43 and also Andreotti et al.44, have taken Eqs. 9 and 7 to predict phase behaviour and flow properties. Their resulting analyses of granular fronts captures many of the main features of granular flows. However, as yet, there is no underlying explanation for these seemingly simple relationships.

\section{INITIATION AND CESSATION OF FLOW}

We have also studied the initiation and cessation of flow, something difficult to probe experimentally. For these studies we used large systems, $A=200 d \times 10 d=2000 d^{2}$ and $h_{\circ} \approx 50 d$, providing better resolution for these processes. In addition, any size effects due to thin samples do not complicate our observations.

We have previously studied initiation of flow from a crystalline static state in $2 \mathrm{D} 22$. After such a system is tilted, a disordering front propagates upwards from the bottom of the pile, melting the ordered structure, until the entire pile is disordered and flows in its steady state form. Although the starting state in $3 D$ need not be ordered, we find that flow initiation in $3 D$ also undergoes a similar bottom-up process.

Initiation was studied for a disordered, static packing of our larger system that was tilted from $\theta=0^{\circ}$ to $24^{\circ}$. See Fig. 13. At the instant of flow initiation, the system slips at the bottom boundary and begins to flow as a plug as indicated in Fig. 13(a). Particles closest to the base undergo collisional motion with the fixed bed leading to the creation of a granular temperature front, as shown in Fig. 13(b). This thermalization front propagates up the pile, disrupting the plug region. After the original initiation slippage, the slip region disappears and the velocity profile appears approximately linear with height, with a crossover to the plug region. After the plug phase melts, the system dilates slightly, leading to a velocity profile of the Bagnold steady state form.
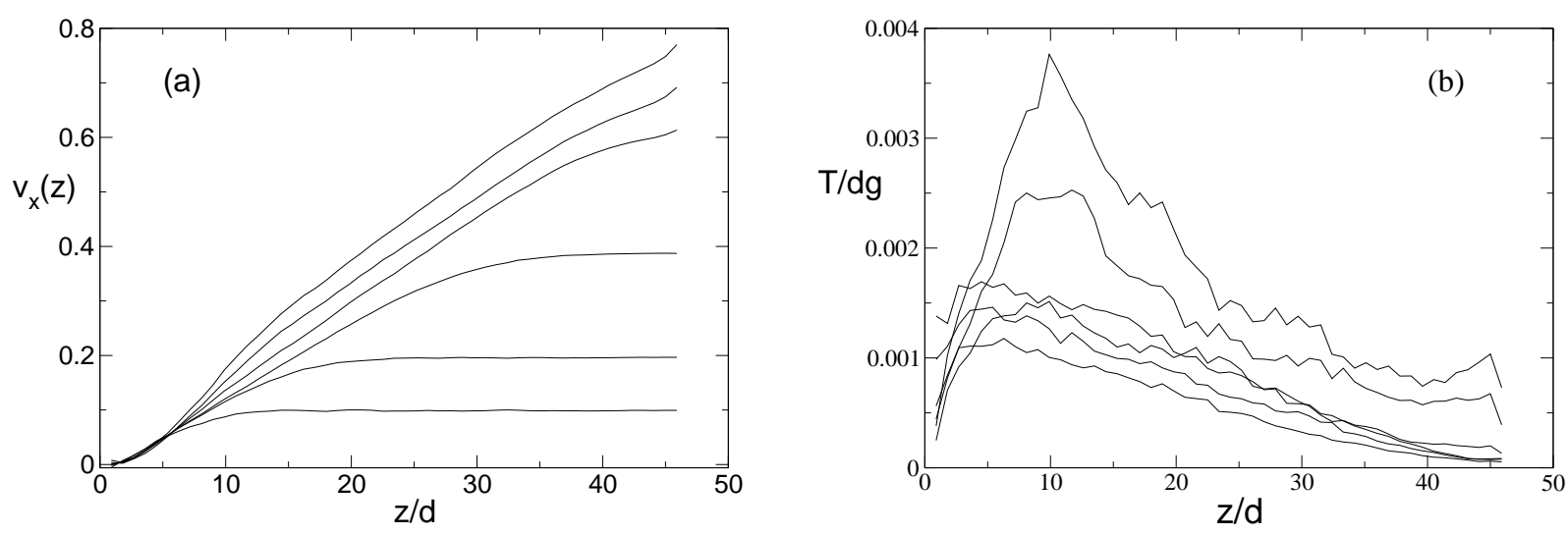

FIG. 13. Initiation of flow: time evolution of the depth profiles of, (a) the velocity in the direction of flow and (b) the granular temperature in the direction of flow, at the initiation of flow for a static state $(h \approx 50 d)$, initially at $\theta=0^{\circ}$, that is tilted to $24^{\circ}$. Initial failure occurs near the base, the pile then "heats up" from the bottom of the pile, a thermalization front propagates up the pile with time, disrupting the plug flow above it. The lowest curve starts at $t=025 \tau$ after the system is first tilted, and we plot curves for $t=0.50 \tau, 1 \tau, 2 \tau, 3 \tau$, and $4 \tau$ respectively. 
Cessation was observed for a system that was initially in steady state flow at $\theta=24^{\circ}$, as the inclination angle was reduced to $\theta=18^{\circ}$, which is below $\theta_{r}$. See Fig. 14. Although it may seem intuitive to expect an exact reversal of the initiation process, this is not observed. After $\theta$ is reduced to below the angle of repose, particles at the bottom of the pile, which have the smallest velocities, come to rest first becoming trapped by the rough base. The frozen layer then serves to trap the slowly moving particles above it. This trapping front propagates up the pile towards the free surface, as indicated in Fig. 14(a). A consequence of this is that the granular temperature cools from the chute base as the whole system gradually comes to rest. We note the emergence of a thin saltating region at the free surface during the cooling process.
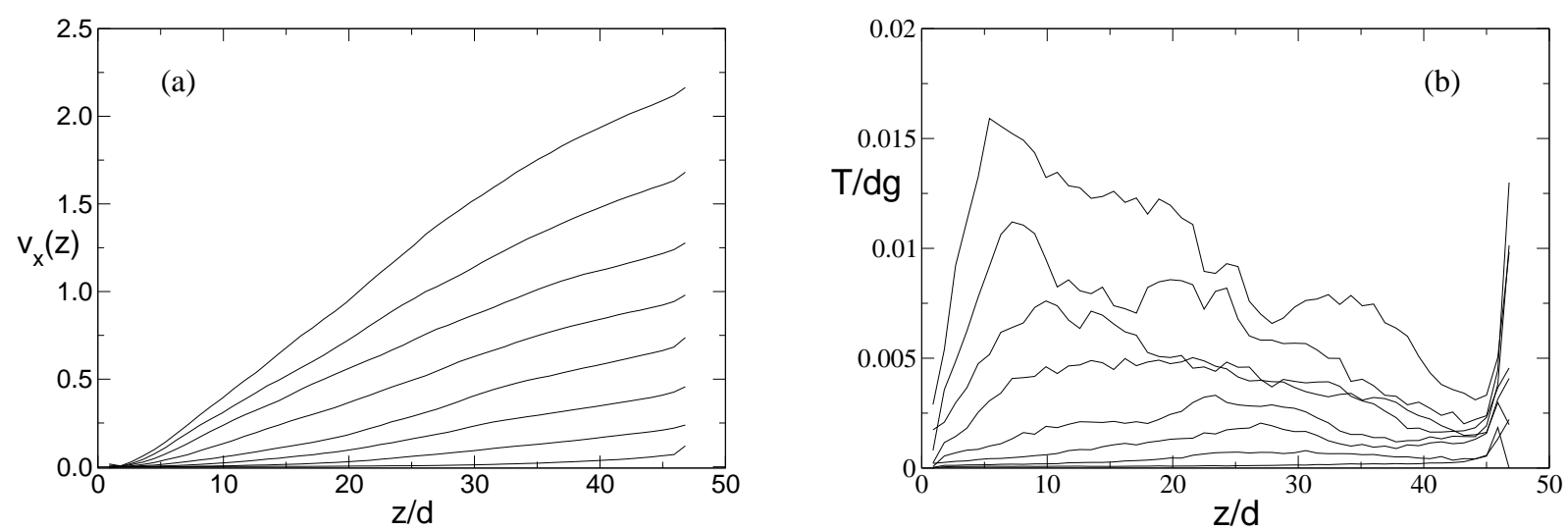

FIG. 14. Cessation of flow: time evolution of the depth profiles of, (a) the velocity in the direction of flow and (b) the granular temperature in the direction of flow, for the cessation of flow of a system with $h \approx 50 d$. Initially in steady state flow with $\theta=24^{\circ}$, the inclination angle is then reduced to $18^{\circ}<\theta_{r}$ leading to the gradual stopping of flow into a jammed state. Particles moving slowest at the base stop flowing first, leading to a freezing front that propagates up the pile. The top curve is taken at a time $100 \tau$ after the angle is first reduced to below the angle of repose, and subsequent curves are separated by $10 \tau$ later.

During this trapping process the pile exhibits two distinct behaviors separated by the trapping front. Below the trapping front, the particles are frozen into a pack, whereas above the trapping front, particles continue to flow. As the trapping front moves up the pile, the depth of the flowing section continuously shrinks with time. As shown in Fig. 14(a), this leads to a bulk velocity profile that consequently transforms from Bagnold-like flow to linear with depth until flow ceases. Essentially, flow cessation is a transient version of route B in Fig. 3 for flow at one angle over a range of flow heights.

Another feature of flow transitions is in understanding the stress response of the system upon initiation and cessation of flow. Classical treatments of granular media国 demand that failure will only occur if the ratio of the shear stress to the normal stress equals the tangent of the angle of repose,

$$
\sigma_{x z}=\sigma_{z z} \tan \theta_{r} .
$$

However, Eq. 10 needs to be modified to take into account the history dependence of the starting point of flow for 
initiation at $\theta_{m}$, and the general stopping point of flow for cessation at $\theta_{r}$. In Fig. 15 the stress ratio $\sigma_{x z} / \sigma_{z z}$ is plotted as a function of inclination angle $\theta$ for initiation of flow (circles) and cessation of flow (squares). We see that $\sigma_{x z} / \sigma_{z z}$ is linear with $\tan \theta$. Still, to observe flow from initiation, the stress ratio must exceed $\tan \theta_{m}$, or for flow to cease its value must be reduced to below $\tan \theta_{r}$. In other words, the shear stress can have a non-zero value and remain static as long as the stress ratio is smaller than the tangent of $\theta_{r}$ or $\theta_{m}$. The particular stress state of the static system is very much history dependent and comes about from the imposed conditions at the instant the system became static 21 .

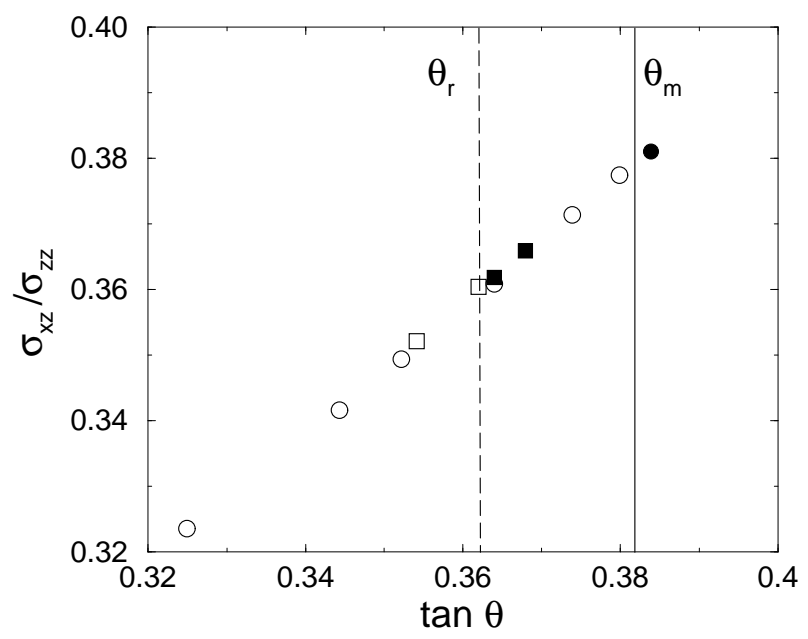

FIG. 15. Ratio of the shear stress to the normal stress $\sigma_{x z} / \sigma_{z z}$ as a function of the tangent of the angle of inclination $\theta$ for initiation of flow (squares) and cessation of flow (circles). The open symbols correspond to static states and the filled symbols are flowing. The lines indicate the positions of $\theta_{r, m}$. The stress ratio is linear with $\tan \theta$, but flow will not occur unless the stress ratio remains above $\tan \theta_{r}$ in the case of a system already flowing, or exceeds $\tan \theta_{m}$ for initiation of flow.

\section{CONCLUSIONS}

We have performed a systematic simulation study of inclined plane, gravity-driven flow of granular materials, elucidating the reasons behind the discrepancies that exist between several different experimental procedures. These arise as a consequence of how far the system is from jamming, or how close the inclination angle of the pile is from the angle of repose.

We have accurately determined the flow phase diagram for a system with a particular interaction parameter set, indicating the height dependence of the angle of repose and the maximum angle of stability. We have also determined the boundary between steady state and unstable flow and find that the maximum angle for steady state flow also exhibits height dependence. This suggests the existence of an intrinsic length scale in granular flows and forms part of our work in progress.

For thick enough piles flowing on a rough inclined plane, say $h \gtrsim 20 d$, the velocity profiles and rheology follow Bagnold scaling. As one reduces the height of the flowing pile, a continuous transition from Bagnold rheology to linear velocity profiles through to avalanche-like dynamics occurs, until finally, one reaches the angle of repose $\theta_{r}$, and flow ceases. As a result, one can obtain various velocity profiles and flow behaviors not only through changing the height of the flowing pile, but also by varying the inclination angle of the chute, such that there is an overlap region 
where one can obtain similar flow properties through either procedure. These features can be used in surface flow treatments 46 . 47 to better predict the evolution of an avalanche surface.

Our investigation on the initiation and cessation of flow shows that the initiation process starts from failure at the base that results in a "heat" front that propagates up the pile. Upon cessation of flow, an effective freezing front propagates up the pile with particles becoming trapped in the frozen layer until the whole system comes to rest. In some cases, flow initiation or cessation only occurred after many time units (approximately equivalent to 20 seconds for a $1 \mathrm{~mm}$ sized particle). A consequence of these findings is that experimental reports of various flow behaviour in flowing piles may also be due to the time window of observation. In general, we have captured all the major observations from experiment, including quantitative agreement of the scaling parameter $\beta$, Eq. 9, from Pouliquen's empirical theory $\mathrm{Q}$.

\section{ACKNOWLEDGEMENTS}

We thank Deniz Ertas and Thomas Halsey for helpful discussions and a critical reading of the manuscript. LES would also like to thank M. A. Aguirre for insightful discussions. This work was supported by the Division of Materials Science and Engineering, Office of Science, U.S. Department of Energy. Sandia is a multiprogram laboratory operated by Sandia Corporation, a Lockheed Martin Company, for the United States Department of Energy under Contract DE-AC04-94AL85000.

${ }^{1}$ S. B. Savage, "Gravity flow of cohesionless granular materials in chutes and channels," J. Fluid Mech. 92, 53 (1979).

${ }^{2}$ C. S. Campbell, C. E. Brennen, and R. H. Sabersky, "Flow regimes in inclined open-channel flows of granular materials," Powder Tech. 41, 77 (1985).

${ }^{3}$ M. Sadjadpour and C. S. Campbell, "Granular chute flow regimes: mass flowrates, flowrate limits and clogging," Advanced Powder Technol. 10, 175 (1999).

${ }^{4}$ H. Ahn, C. E. Brennen, and R. H. Sabersky, "Analysis of the Fully Developed Chute Flow of Granular Materials," J. Appl. Mech. 59, 109 (1992).

${ }^{5}$ P. C. Johnson, P. Nott, and R. Jackson, "Frictional-collisional equations of motion for particulate flows and their application to chutes," J. Fluid Mech. 210, 501 (1990).

${ }^{6}$ T. G. Drake, "Structural features in granular flows," J. Geophysical Res. 95, 8681 (1990).

${ }^{7}$ T. Pöschel, "Granular material flowing down an inclined chute: a molecular dynamics simulation,” J. Phys. II 3, 27 (1993).

${ }^{8}$ X. M. Zheng and J. M. Hill, "Molecular dynamics modelling of granular chute flow: density and velocity profiles," Powder Tech. 86, 219 (1996).

${ }^{9}$ O. Pouliquen, "Scaling laws in granular flows down rough inclined planes," Phys. Fluids 11, 542 (1999). 
${ }^{10}$ E. Azanza, F. Chevoir, and P. Moucheront, "Experimental study of collisional granular flows down an inclined plane," J. Fluid Mech. 400, 199 (1999).

${ }^{11}$ C. Ancey, "Dry granular flows down an inclined channel: Experimental investigations on the frictional-collisional regime," Phys. Rev. E 65, 011304 (2001).

12 P.-A. Lemieux and D. J. Durian, "From avalanches to Fluid Flow: A continuous picture of grain dynamics down a heap," Phys. Rev. Lett. 85, 4273 (2000).

${ }^{13}$ T. S. Komatsu, S. Inagaki, N. Nakagawa, and S. Nasuno, "Creep motion in a granular pile exhibiting steady surface flow," Phys. Rev. Lett. 86, 1757 (2001).

${ }^{14}$ D. V. Khakhar, A. V. Orpe, P. Adresen, and J. M. Ottino, "Surface flow of granular materials: model and experiments in heap formation," J. Fluid Mech. 441, 255 (2001).

${ }^{15}$ C. Ancey and P. Evesque, "Frictional-collisional regime for granular suspension flows down an inclined channel," Phys. Rev. E 62, 8349 (2000).

${ }^{16}$ P. Mills, D. Loggia, and M. Tixier, "Model for a stationary dense granular flow along an inclined wall," Europhys. Lett. 45, $733(1999)$.

${ }^{17}$ L. Bocquet, W. Losert, D. Schalk, T. C. Lubensky, and J. P. Gollub, "Granular shear flow dyanmics and forces: Experiment and continuum theory," Phys. Rev. E 65, 011307 (2001).

${ }^{18}$ I. S. Aranson and L. S. Tsimring, "Continuum description of avalanches in granular media," Phys. Rev. E 64, 020301 (2001).

${ }^{19}$ O. R. Walton, "Numerical simulation of inclined chute flows of monodispers, inelastic, frictional spheres," Mech. Mat. 16, 239 (1993).

${ }^{20}$ D. M. Hanes and O. R. Walton, "Simulations and physical measurements of glass spheres flowing down a bumpy incline," Powder Tech. 109, 133 (2000).

${ }^{21}$ D. Ertaş, G. S. Grest, T. C. Halsey, D. Levine, and L. E. Silbert, "Gravity-driven dense granular flows," Europhys. Lett. 56, $214(2001)$.

${ }^{22}$ L. E. Silbert, D. Ertaş, G. S. Grest, T. C. Halsey, D. Levine, and S. J. Plimpton, "Granular flow down an inclined plane: Bagnold scaling and rheology," Phys. Rev. E 64, 051302 (2001).

${ }^{23}$ R. A. Bagnold, "Experiments on a Gravity-Free Dispersion of Large Solid Spheres in a Newtonian Fluid under Shear," Proc. R. Soc. London Ser. A 255, 49 (1954).

${ }^{24}$ R. A. Bagnold, "The Flow of Cohesionless Grains in Fluid," Proc. Roy. Soc. A 249, 235 (1956).

${ }^{25}$ M. Y. Louge and S. C. Keast, "On dense granular flows down flat frictional inclines," Phys. Fluids 13, 1213 (2001).

${ }^{26}$ L. E. Silbert, G. S. Grest, S. J. Plimpton, and D. Levine, submitted to Phys. Fluids (unpublished).

${ }^{27}$ M. A. Aguirre, N. Nerone, A. Calvo, I. Ippolito, and D. Bideau, "Influence of the number of layers on the equilibrium of a granular packing," Phys. Rev. E 62, 738 (2000). 
28 A. Daerr and S. Douady, "Two types of avalanche behaviour in granular media," Nature 399, 241 (1999).

${ }^{29}$ O. Pouliquen and N. Renaut, "Onset of Granular Flows on an Inclined Rough Surface: Dilatancy Effects," J. Phys. II 6, $923(1996)$.

${ }^{30}$ High polydispersity may lead to such effects as segregation.

${ }^{31}$ O. R. Walton and R. L. Braun, "Viscosity, Granular-Temperature, and Stress Calculations for Shearing Assemblies of Inelastic, Frictional Disks," J. Rheo. 30, 949 (1986).

${ }^{32}$ P. A. Cundall and O. D. L. Strack, "A discrete numerical model for granular assemblies," Géotechnique 29, 47 (1979).

${ }^{33}$ We note that in this study we are modelling Hertzian contacts with static friction and tangenetial velocity damping. In our previous study most of the 3D results were for linear spring contacts with static friction only. In the case of Hertzian contacts, because the coefficient of restitution is velocity dependent and goes to 1 as $\mathbf{v}_{n} \rightarrow 0$, the rolling damping term $\gamma_{t}$ ensures that the energy of a static pile goes to zero.

${ }^{34}$ A. Daerr, "Dynamical equilibrium of avalanches on a rough plane," Phys. Fluids 13, 2115 (2001).

${ }^{35}$ P. Mills, M. Tixier, and D. Loggia, "Influence of roughness and dilatancy for dense granular flow along an inclined wall," Eur. Phys. J. E 1, 5 (2000).

${ }^{36}$ A. Mehta and G. C. Barker, "Bistability and hysteresis in tilted sandpiles," Europhys. Lett. 56, 626 (2001).

${ }^{37}$ L. E. Silbert, D. Ertaş, G. S. Grest, T. C. Halsey, and D. Levine, "Geometry of frictional and frictionless sphere packings," Phys. Rev. E 65, 031304 (2002).

${ }^{38}$ M. A. Aguirre, N. Nerone, I. Ippolito, A. Calvo, and D. Bideau, "Granular packing: influence of different parameters on its stability," Gran. Matter 3, 75 (2001).

${ }^{39}$ N. Nerone, M. A. Aguirre, A. Calvo, I. Ippolito, and D. Bideau, "Surface fluctuations in a slowly driven granular system," Physica A 283, 218 (2000).

${ }^{40}$ Y. C. Zhou, B. H. Xu, and A. B. Yu, "Numerical investigation of the angle of repose of monosized spheres," Phys. Rev. E 64, 021301 (2001).

${ }^{41}$ L. E. Silbert, D. Ertaş, G. S. Grest, T. C. Halsey, and D. Levine, "Analogies between granular jamming and the liquid-glass transition," Phys. Rev. E 65, 051307 (2002).

42 M. D. Silva and J. Rajchenbach, "Stress transmission through a model system of cohesionless elastic grains," Nature 406, $708(2000)$.

${ }^{43}$ O. Pouliquen, "On the shape of granular fronts down rough inclined planes," Phys. Fluids 11, 1956 (1999).

${ }^{44}$ B. Andreotti, A. Daerr, and S. Douady, "Scaling laws in granular flows down a rough plane," Phys. Fluids 14, 415 (2002).

${ }^{45}$ R. M. Nedderman, Statics and Kinematics of Granular Materials (Cambridge University, Cambridge, 1992).

${ }^{46}$ A. Aradian, E. Raphael, and P.-G. de Gennes, "Thick surface flows of granular materials: Effect of the velocity profile on the avalanche amplitude," Phys. Rev. E 60, 2009 (1999). 
${ }^{47}$ S. Douady, B. Andreotti, and A. Daerr, "On granular surface flow equations," Eur. Phys. J. B 11, 131 (1999). 